\title{
Flow Features of Three Side-by-side Circular Cylinders at Low Reynolds Number
}

\author{
Liu Junkao, Hao Yaqiang, Dong Dibo, Chen Weishan \\ State Key Laboratory of Robotics and System, Harbin Institute of Technology, Harbin, Heilongjiang Province, China
}

\begin{abstract}
In order to study the fluctuation of kinetic parameter of cylinder matrix in incompressible stationary flow, the flow fluid around three side-by-side circular cylinders are simulated using Immersed BoundaryLattice Boltzmann method (IB-LBM). Drag and lift force of the three cylinders are investigated as the interval between each cylinder varied from zero to five times of the cylinder diameter. Five flow patterns are defined according to the vortices structure in the downstream of the cylinders. Power spectrum analysis of lift force is developed to explain the vortex patterns. Through the research, we find the strength and phase of the gap flow play an important role in the vortex formatting process. The vortices shedding from different cylinders neutralize and combine in the near wake, contributing a lot to the variation of forces.
\end{abstract}

\section{Introduction}

The interaction between solid and fluid, which has much significance in aviation, energy harvesting and equipment manufacturing, has attracted many attentions these years. Flowing past bluff body is the most common model in dealing with this kind of research, because of its wide existence, for example, power transmission lines, offshore work platform, bridge pier, etc. Wake structure interaction, vortex shedding and jet flow are the main characteristics of this kind of flow [1]. Many methods, based on the theory study, experiment and numerical simulation, have been developed to make the flow patterns of multiple cylinders in steady flow clear. Experiment study starts in an earlier time. Lift and drag coefficients, frequency of vortex shedding, pressure around the cylinders, as well as the wake model, are the main factors helping us to comprehend the nature of the flowing.

Previously, many people have done a very significant job. Surface pressure measurements, drag and lift forces measurement and the wake observation give us two explicit knowledge [2-5]. One is the flow patterns of the wake behind two side-by-side circular cylinders include three main regimes as the interval (T/D) increasing. When $\mathrm{T} / \mathrm{D}<1.2$, the two cylinders act as single one forming one single street; when $1.2<\mathrm{T} / \mathrm{D}<2.0$, the flow interplay and the wake are asymmetrical; for $\mathrm{T} / \mathrm{D}>2.0$, the coupled streets occurs. The other knowledge is the periodic vortex-shedding contribute to the fluctuated forces on cylinders. Vortex-shedding changes the distribution of hydraulic pressure, and the cylinders are subjected to a lift and drag force as a result. The fluctuation of lift force synchronizes to the vortex shedding.

Till now, to find out the nature of fluid and structure, many experiments are still undertaken with some new technology. The hot-wire, laser-induced fluorescence flow visualization and Particle Image Velocimetry (PIV) techniques are usually used to examine the flow filed, while fiber grating and piezo-electric technologies are employed to measure the dynamic features. And the sharp and configurations of cylinders also are settled in a more complicated way [6, 7]. Alam and Zhou identified four flow regimes of flow behind two side-by-side square cylinders using flow visualization method and the rules of street-merging were discussed [8]. The investigation presents similar but different results. The flow around four cylinders in a square configuration is observed by Lam and Chan in 2003 using laser-induced fluorescence (LIF) visualization and particle image velocimetry (PIV). The transformation of flow patterns were investigated as the angle incident varying from $\alpha=0^{\circ}$ to $45^{\circ}$. Like two side by side cylinders, the wake also is classified to three flow regimes according to $\alpha$. Meanwhile, the configuration of cylinders leads to strong vortex-induced vibration at $\alpha=15^{\circ}$ because of the jet flow between the near wake of upstream cylinder and downstream cylinder [9]. The flow filed around three side-by-side cylinders was investigated by Zhang and Zhou in 2001, arming to find out the effect of different interval on the flow patterns. A comparison between equal and unequal cylinder spacing was made on pressure, drag and lift coefficient, and vortex formation mechanisms [10].

Numerical simulation is a suppositional way to imitate the flow field significantly. Meneghini and Saltara carried out computations for flow interference between 
two circular cylinders in abreast and tandem arrangement for $\mathrm{Re}$ ranging from 100 to 200 through the finiteelement method. The study presented vorticity around the cylinders and force evolution with time [11]. Unsteady three-dimensional flow simulations were implemented on different gap/diameter ratios. The growth of vertical structures and different modes of flow bifurcation along their spanwise extended core lines were discussed [12]. As to square cylinders, Burattini and Agrawal computed the two-dimensional flow field around two side-by-side square cylinders while the lateral separations between the cylinders $\mathrm{T} / \mathrm{D}$ ranges from 0.5 to 6 . Three regimes are measured during the varying of intervals, in-phase, inantiphase and quasi-periodic, which has been confirmed by experiment [13]. Supradeepan classified the flow pattern more minutely. Five different flow patterns were found for the flow behind two side-by-side cylinders through periodic variation of flow, frequency spectrum analysis and phase diagrams [14].

There are few researches on three circular cylinders arranged side-by-side. This paper focus on the parameters fluctuating and the flow regime of the flow field around three circular cylinders arranged side-by-side. Immersed Boundary-Lattice Boltzmann Method is used to compute the fluid date. The relationship between fluid regime and lift force of middle cylinder are discussed.

\section{Numerical simulation method - LBM}

Unlike other common numerical system focused on molecular dynamics or macro-kinetics, Lattice Boltzmann method utilizes dispersed lattice and dispersed time based on microscopic models as well as macrokinetics equations, taking macro-scope motion as the response of the collective behavior of the dispersed particles [15]. LBM takes the space and time as dispersed regular lattices, the fluid as dispersed particles. Particles' movement is restricted by lattices in particular rules. The motions of particle are consist of two progress, collision and propagation, each of which stands for the dynamics and kinematics progress. Microscopic statistics method is also used to get macro-scope parameters, like velocity and pressure.

The use of macro-scope dynamics and microscopic statistics on the dispersed microscope model provides many advantages to LBM, which involve the clear operating process, convenient dispose of complicated boundary conditions and easy access to parallel algorithms. As the development of parallel computer technics, LBM gets more attention those years.

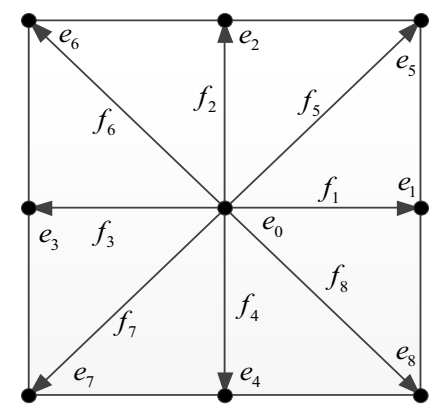

Figure 1. D2Q9 model
Different dispersed-model stands for different ways of particles collision and propagation, whereas all of them obey the law of mass and momentum conservation. In this paper, we use D2Q9 model [16], where D means the space dimensions and $\mathrm{Q}$ means the number of particles. The model of D2Q9 is shown in Fig. 1.

The evolution equation of LBM dispersed by twodimensional viscous incompressible D2Q9model is as follow:

$$
f_{i}\left(x+c_{i} \Delta t, t+\Delta t\right)-f_{i}(x, t)=\Omega_{i}
$$

where:

$$
\begin{gathered}
\Omega_{i}=-\frac{f_{i}(x, t)-f_{i}^{e q}(x, t)}{\tau} \\
f_{i}^{e q}=\rho \omega_{i}\left[1+\frac{\boldsymbol{c}_{i} \boldsymbol{u}}{c_{s}^{2}}+\frac{\left(\boldsymbol{c}_{i} \boldsymbol{u}\right)^{2}}{2 c_{s}^{2}}+\frac{\boldsymbol{u}^{2}}{2 c_{s}^{2}}\right] \\
\boldsymbol{c}_{i}= \begin{cases}(0,0) \boldsymbol{c} & i=0 ; \\
\left(\cos \frac{i-1}{2} \pi, \sin \frac{i-1}{2} \pi\right) \boldsymbol{c} & i=1,2,3,4 ; \\
\left(\sqrt{2} \cos \left(\frac{i-5}{2}+\frac{1}{4}\right) \pi, \sqrt{2} \sin \left(\frac{i-5}{2}+\frac{1}{4}\right) \pi\right) \boldsymbol{c} & i=5,6,7,8 .\end{cases}
\end{gathered}
$$

where $i$ is the direction of discrete velocity, $i=0,1,2 \ldots 8$; $\Delta t$ refers to the discrete time step; $f_{i}(x, t)$ is the particle velocity distribution functions, defined on the particle location $x$ and time $t ; \tau$ is the single-relaxation-time parameter which controls the rate of approach to equilibrium; $\boldsymbol{c}_{i}$ represents the particle velocity in direction; $\Omega_{i}$ is the collision operator, which stands for the rate of $f_{i}(x, t)$ after collision; $f_{i}^{e q}$ is expressed as the local equilibrium distribution function; $\rho$ and $\boldsymbol{u}$ are the macroscope density and velocity; $\boldsymbol{c}_{s}=\boldsymbol{c} / \sqrt{3}$ is the sound speed; while $\omega_{i}$ is weight coefficient, $\omega_{0}=4 / 9, \omega_{i}=1 / 9(\mathrm{i}=1,2,3.4)$, $\omega_{i}=1 / 36(i=5,6,7,8)$.

The macro-scope velocity and momentum are defined as follows in D2Q9 model.

$$
\rho=\sum_{i} f_{i}=\sum_{i} f_{i}^{e q}, \rho \boldsymbol{u}=\sum_{i} f_{i} \boldsymbol{c}_{i}=\sum_{i} f_{i}^{e q} \boldsymbol{c}_{i}
$$

This paper takes those three cylinders (with equal diameters of 25) and the flow field (the size is $1400 \times 800$ ) as research objects. As showing in Fig. 2, take the left bottom of flow field as the origin of coordinates, the flow and cross flow direction as $x$ and $x$ axis. The initial and boundary conditions must be specified in advance. The three cylinders are named $\mathrm{C} 1, \mathrm{C} 2$ and $\mathrm{C} 3$ in sequence.

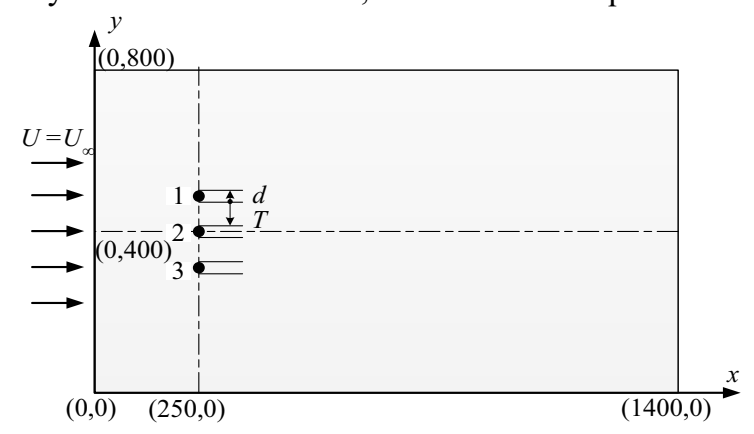

Figure 2. Numerical region and boundary conditions 
where $d$ means the cylinders diameter; $u, v$ is the velocity on $x, y$ directions; $\mathrm{Re}=u d / v$ represents the Reynolds number, $v$ is kinematic coefficient of viscosity; $T$ stands for interval coefficient where $T=D / d$.

When time $t=0$, the flow field is assumed to be static and a uniform and constant free-stream flow is introduced into the domain from left boundary after that. Upper and lower boundaries are disposed with non-equilibrium extrapolation scheme to eliminate the impact on the flow regime. Non-slip boundary condition is invoked to all cylinder boundaries. Considering the significance role of the lattice structure in the numerical model, Immersed Boundary Method is applied to the cylinder boundaries. The boundary conditions are as follows:

Inlet: $u=U_{\infty}, v=0$

Outlet: $\partial u / \partial x=\partial v / \partial x=0$

Upper and lower boundary: $\partial u / \partial y=0, v=0$

Cylinder boundary: $u=v=0$.

In this paper, the interval ranges from 0 to 5 . We get the conclusion on the influence of interval to flow pattern, and interaction between fluid and solid structure, through the analysis of the static condition parameters, like the lift, drag force coefficient and vortex structure. Furthermore, in order to reveal the nature of vortex shedding of the three cylinders, FFT algorithm is developed to analysis the vortex shedding frequency.

\section{Results and discussion}

In this paper, the flow behavior around three circular cylinders arranged side-by-side with the same interval at $\operatorname{Re}($ Reynolds number $)=80$ and 100 are investigated. The mean drag and the VPP lift force of $\mathrm{C} 2$ are shown in Fig. 3. The statistic value is compared with that of a single cylinder.

From the chart we can find both the lift and drag force reaches the maximum value when $\mathrm{T}=0$. The drag coefficient decrease step by step when $\mathrm{T}>0$, reaching a valley when $\mathrm{T}=1.0 . \mathrm{T}=1.0$ is a peak with a value a little higher than $\mathrm{T}=0.5$. The drag coefficient decrease slightly with the value inching closer to the value for a single cylinder. As to the lift coefficient, there is a sharp decrease on lift coefficient when $\mathrm{T}=0.5$ with the value evidently smaller than on single cylinder. After that, the value increases, reaching the peak twice when $T=1$ and $\mathrm{T}=3.5$. When $\mathrm{T}>3.5$, the lift force decrease step by step, approaching the value of one single cylinder. To explain the fluctuation with $\mathrm{T}$, the flow regime is investigated. Five flow patterns are defined according to the vorticity contours and the fluctuating of mean drag and VPP lift force.

Referring to the review the predecessors of advances [16], the five defined patterns includes the single solid model, interim model, in-phase model, diff-phase model and separate model. Meanwhile, contrast to $\operatorname{Re}=80$, the certain pattern condition occurs at a smaller interval coefficient $\mathrm{T}$ at $\mathrm{Re}=100$.

As shown in Fig. 4, the flow pattern behind three side-by-side cylinders performs as the single model when $\mathrm{T}=0,0.5$ at $\mathrm{Re}=80$ and 100 . When $\mathrm{T}=0$, there is no fluid flowing through the cylinder gaps. The vortex shedding from these cylinders is rounded and periodic as shown in Fig. 4 (a). When $\mathrm{T}=0.5$, the interval is so narrow that the vortex from the gaps of these three cylinders are much weaker than these from the top of $\mathrm{C} 1$ and the bottom of C3, which we can called the main-vortices. There is no obvious vortex shedding in the gap flow. As the Fig. 4 (b) showed, the main-vortices are spindly in the near weak but rounded and regular and in the far wake, similar to the single cylinder wake.

From the Fig. 4 (b) we may get the cause of the sharp decrease when $T=0.5$. As shown, the interplay between the vortices from the gaps and the main-vortices is evident in the near wake. Part of the negative vortex from the top of $\mathrm{C} 2$ and the positive vortex from the bottom of $\mathrm{C} 1$ neutralize in a limited region and the remanent parts with the same polarity trend to combine together forming single larger vortex street on the action of hydrodynamics.
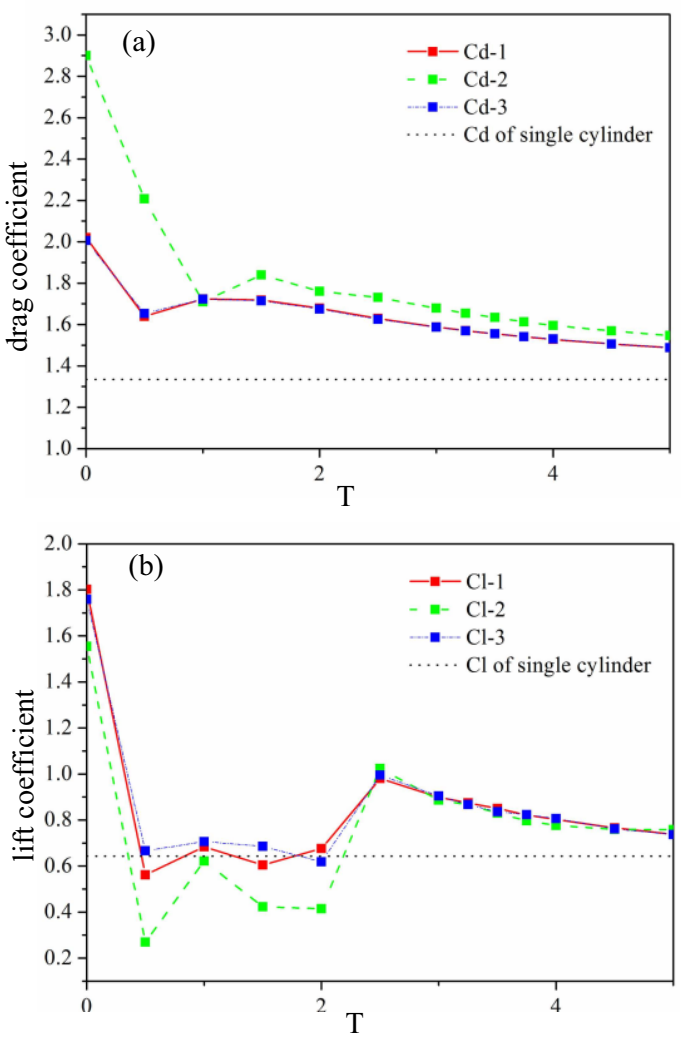

Figure 3. The fluctuating of mean drag (a) and VPP lift (b) force of $\mathrm{C} 2$ with $\mathrm{T}$ at $\mathrm{Re}=100$

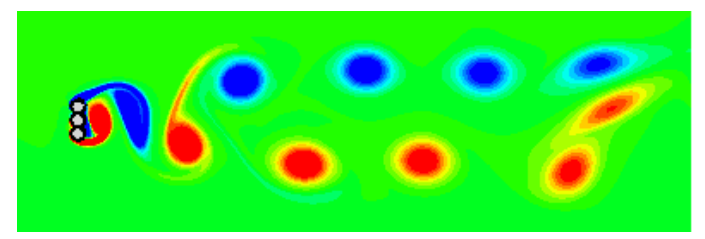

(a) $\mathrm{Re}=100 \mathrm{~T}=0$

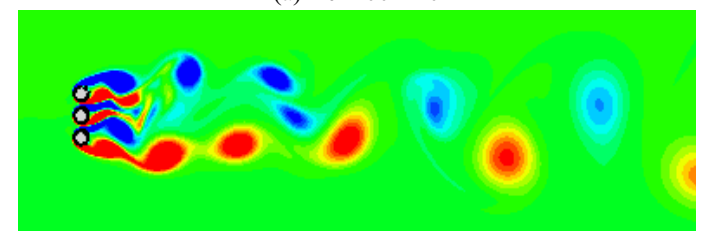

(b) $\mathrm{Re}=100 \mathrm{~T}=0.5$

Figure 4. The vorticity contours for interval coefficients when $\mathrm{T}=0$ and $\mathrm{T}=0.5$ at $\mathrm{Re}=100$ 
Fig. 5 shows the pattern of interim model of three circular cylinders arranged side-by-side, which are computed when $\mathrm{T}=1.0,1.5$ at $\mathrm{Re}=80$ and $\mathrm{T}=1.0$ at $\mathrm{Re}=100$. The spindly vortex fragmented when flowing away from the cylinders before combined, forming many irregular and aperiodic less-intense vortices. Many small vortices interact with each other, formatting the chaotic nature of this stage, which is evident in Fig. 5 (b). As the size of cylinders interval keeps growing, the vortex from the gap flow cannot be ignored and the vortex stretches longer in the near wake, resulting in much interference between vortices. For example, the positive vortex from the top of $\mathrm{C} 2$ trends to combine with the main vortex of same sign from the top of $\mathrm{C} 1$ on the action of hydrodynamics, while there is a negative wake on the way.

Furthermore, this kind of disorganized nature of the vorticity field also is observed by Sumner from the PIV experiments [16]. With the comparison of Fig. 5 (a) and Fig. 5 (b), we find the interim model occurs at smaller gap size when Reynolds number is lager.

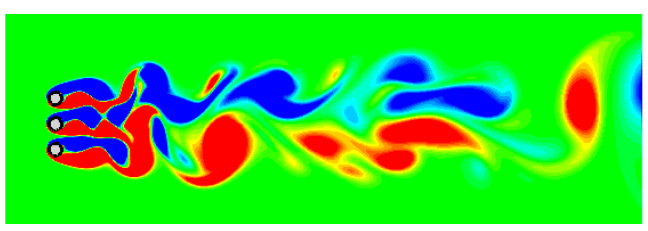

(a) $\operatorname{Re}=80 \mathrm{~T}=1.5$

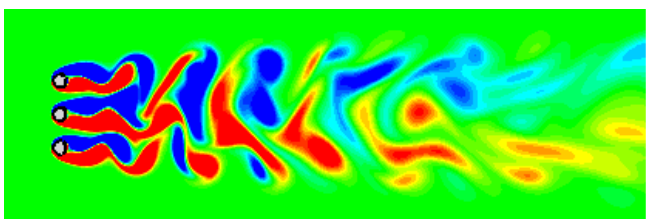

(b) $\mathrm{Re}=100 \mathrm{~T}=1.0$

Figure 5. The vorticity contours for interval coefficients when $\mathrm{T}=1.5$ at $\mathrm{Re}=80$ and when $\mathrm{T}=1.0$ at $\mathrm{Re}=100$

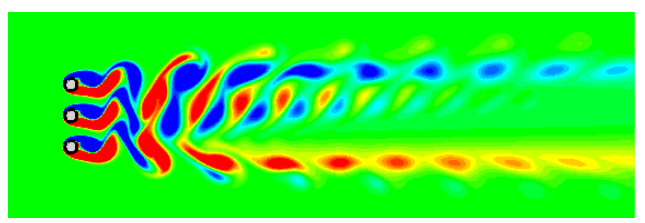

(a) $\operatorname{Re}=80 \mathrm{~T}=2.0$

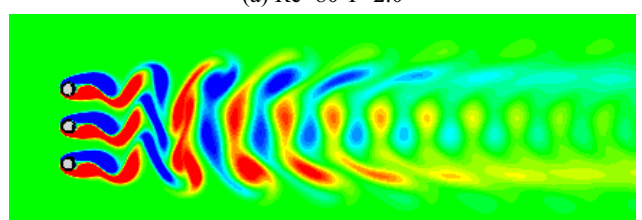

(b) $\mathrm{Re}=100 \mathrm{~T}=1.5$

Figure 6. The vorticity contours for interval coefficients when $\mathrm{T}=1.5$ at $\mathrm{Re}=80$ and when $\mathrm{T}=1.0$ at $\mathrm{Re}=100$

Fig. 6 shows the pattern of in-phase model of three circular cylinders arranged side-by-side, when $\mathrm{T}=2.0$ at $\mathrm{Re}=80$ and $\mathrm{T}=1.5$ and 2.0 at $\mathrm{Re}=100$. In this stage, gap flow vanishes. The vortices flow from the three cylinders shedding in phase with each other in the near cylinder area, which is evident in the time history of force coefficients, as shown in Fig. 8. In the further downstream area of the cylinders, reacting of vortices occurs, resulting in a wide wake. The vortices of opposite sign neutralize and the vortices of same sign combine together. As the distance is beyond a certain value, the interference effect weaker and the flow subside. The wake divides into two parts of the opposite sign, forming two trains of vortices staggered in the water flowing direction, as shown in Fig. 7 (a).

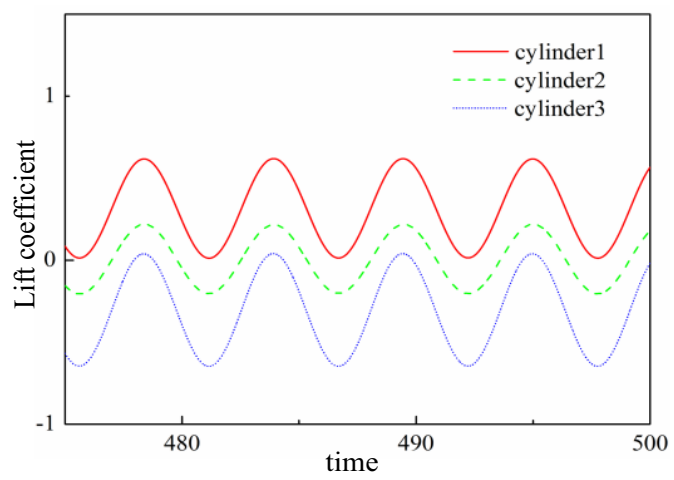

Figure 7. The stable time history of lift and drag coefficient when $\mathrm{T}=1.5$ at $\mathrm{Re}=100$

Fig. 8 shows the pattern of diff-phase of three circular cylinders arranged side-by-side, which is computed when $\mathrm{T} \geq 2.5$. Each of the vortex shedding regimes of the three cylinders is similar to that of the single cylinder, with little interaction between each other in the near weak. Maybe that is why the lift and drag coefficient are close to the single cylinder value.

While in phase, the asynchrony behavior is observed evidently. The vortices shedding from $\mathrm{C} 1$ and $\mathrm{C} 3$ are symmetrically synchronous with opposite phase, while the vortex of center cylinder is a little phase-leading. As shown in Fig. 8 (a) and Fig. 9, the weak of cylinder 3 is anti-phase to cylinder 1 but almost in-phase to C2. Fig. 8 (b) $(\mathrm{D}=3.5)$ indicates, in the far weak of the three cylinders, the vortices with the same sign from $\mathrm{C} 2$ and $\mathrm{C} 3$ combine, forming a string of irregular vortices along the flow direction. Meanwhile, the vortices from $\mathrm{C} 1$ are stable, showing little interaction with the vortices from $\mathrm{C} 2$ or $\mathrm{C} 3$. The vorticity contours of diff-phase matches the experiment of Williamson, revealing that the vortex shedding anti-phase is stable [4]. C1 and C3 have the same vortex shedding frequency according to the frequency spectrum of lift force, as shown in Fig. 11. The vortex shedding of $\mathrm{C} 2$ is a little frequenter, as shown in the Fig. 10.

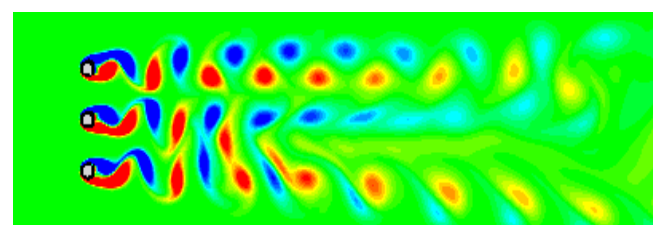

(a) $\operatorname{Re}=100 \mathrm{~T}=2.5$

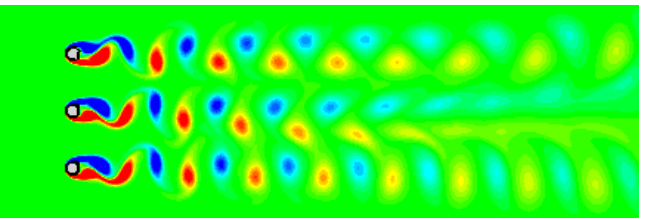

(b) $\mathrm{Re}=100 \mathrm{~T}=3.5$

Figure 8. The vorticity contours for interval coefficients when $\mathrm{T}=1.5$ at $\mathrm{Re}=80$ and when $\mathrm{T}=1.0$ at $\mathrm{Re}=100$ 


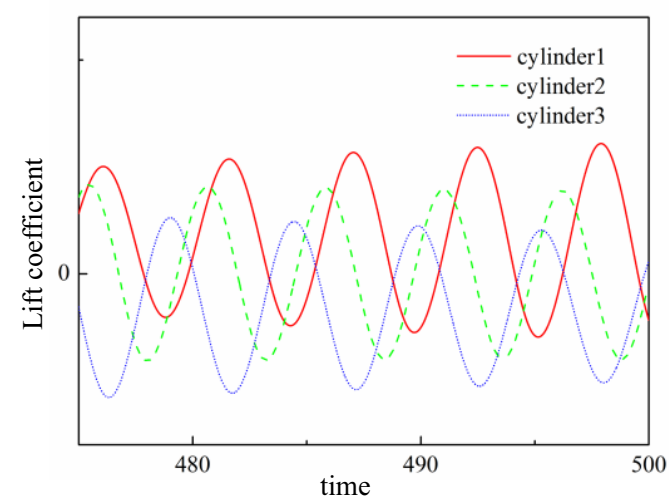

Figure 9. The stable time history of lift coefficient when $\mathrm{T}=2.5$ at $\operatorname{Re}=100$

Fig. 11 shows the pattern of separate model of the three circular cylinders arranged side-by-side, which are computed when $\mathrm{T}=5.0$. The vorticity contour indicates the interaction among the vortices of cylinders can be ignored in the near and far wake. The flow pattern of each cylinder can be seen as the single cylinder flow regime with the same lift and drag coefficient and vortexshedding frequency.

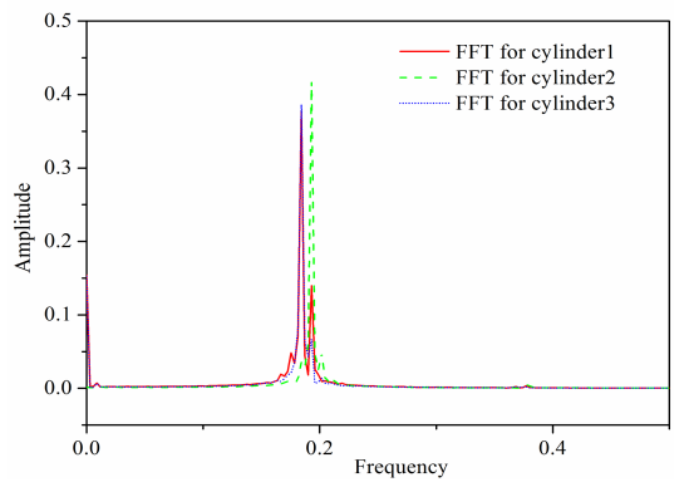

Figure 10. Power spectra analysis of lift coefficient when $T=2.5$ at $\mathrm{Re}=100$

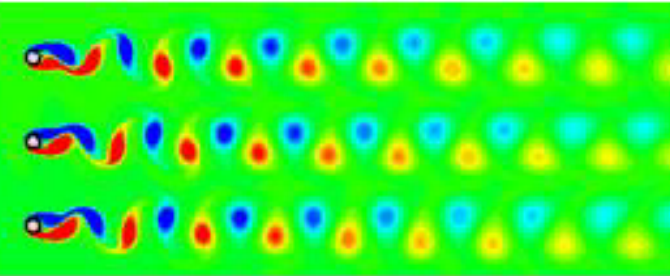

Figure 11. The vorticity contours for interval coefficients when $\mathrm{T}=5.0$ at $\mathrm{Re}=100$

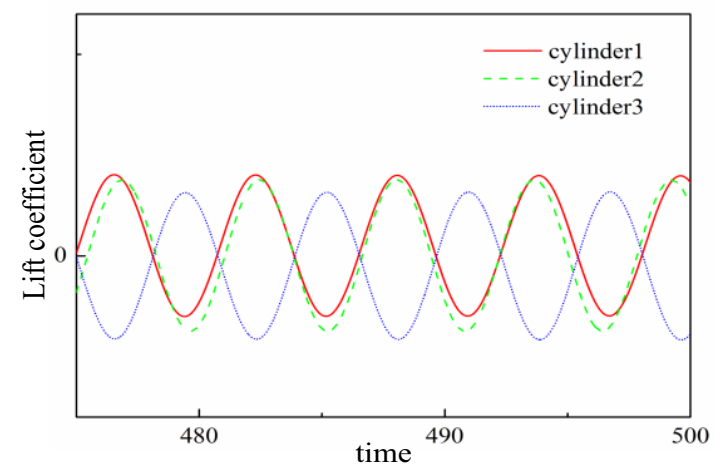

Figure 12. The stable time history of lift and drag coefficient when $\mathrm{T}=1.5$ at $\mathrm{Re}=100$

\section{Conclusion}

Flow behaviors around three circular cylinders arranged side-by-side at low Reynolds number of 80 and 100 are computed using the Immersed Boundary-Lattice Boltzmann Method in two dimensional spaces. The research focuses on the reveal of the nature between the cylinders interval and the flow parameters. The wake structure, lift and drag coefficient and the vortexshedding frequency are investigated.

As the intervals between cylinders increase from 0 to 5.0, five flow patterns (namely the single solid model, interim model, in-phase model, diff-phase model and separate model) are defined, based on the study of vorticity contours of these three paratactic cylinders. The vortex shedding of $\mathrm{C} 1$ and $\mathrm{C} 3$ is synchronous and symmetric, while the vortex shedding of $\mathrm{C} 2$ is a little phase-leading, when the interval coefficient $T \geq 2.5$. In addition, higher Reynolds number leads to a thinner sheer layer, which has the similar consequence with wider interval.

At interim model, there is a sharp decrease in the lift coefficient of $\mathrm{C} 2$, and the gap flow around $\mathrm{C} 2$ is very weak and the neutralization occurs between the positive and negative vortices, while the frequency have little change. This result may be used to reduce the vibration strength of rigid structure in the flowing fluid environment. The interaction between blunt-body and flexible-body requires further discuss.

\section{References}

1. Islam S U, Rahman H, Ying Z C, Journal of the Brazilian Society of Mechanical Sciences and Engineering, 38, pp. 843-858, (2014)

2. Bearman P W, Wadcock A J, Journal of Fluid Mechanics, 61, pp. 499-511, (1973)

3. Zdravkovich M M, Journal of Fluids Engineering, 99, pp 618-633, (1977)

4. Williamson C H K, Journal of Fluid Mechanics, 159, pp 1-18, (1985)

5. Wang Z J, Zhou Y, International journal of heat and fluid flow, 26, pp. 362-377, (2005)

6. Zhou Y, Wang Z J, So R M C, Journal of Fluid Mechanics, 443, pp 197-229, (2001)

7. Lam K, Li J Y, So R M C, Journal of Fluids and Structures, 18, pp 305-324, (2003)

8. Alam M M, Zhou Y, Physics of Fluids, 25, 085106, (2013)

9. Lam K, Li J Y, Chan K T, Journal of Fluids and Structures, 17, pp. 665-679. (2003)

10. Zhang H J, Zhou Y, Physics of Fluids, 13, pp. $3675-$ 3686, (2001)

11. Meneghini J R, Saltara F, Siqueira C L R, Journal of fluids and structures, 15, pp 327-350, (2001)

12. Sau A, Hsu T W, Ou S H, Physics of Fluids, 19, 084105, (2007)

13. Burattini P, Agrawal A, Computers \& Fluids, 77, pp. 134-142, (2013)

14. Supradeepan K, Roy A, Physics of Fluids, 26, 063602, (2014) 
15. Kadanoff L P, Physics today, 39, (1986)

16. Sumner D, Wong S S T, Price S J, Journal of Fluids and Structures, 13, pp 309-338, (1999) 